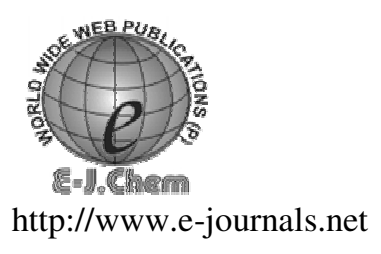

\title{
Spectrophotometric Study of Nitrogen Base Adducts of Nickel(II)-4-methyl-8-quinolinate
}

\author{
T. SURESH, S. SANTOSH KUMAR, N. M . KOTTURESHAWARA, \\ M. REVANASIDAPPA*, SYED KHASIM ${ }^{\$}$ and SURESH $^{\#}$
}

Department of Industrial Chemistry,

Gulbarga University Post Graduate Centre, Bellary- 583104, India.

*Department of Chemistry, ${ }^{\$}$ Department of Physics, PES School of Engineering, Bangalore- 560100, India.

${ }^{\#}$ Department of Chemistry, Bellary Engineering College, Bellary- 583104, India.

revum@ rediffmail.com

Received 29 August 2007; Revised 26 September 2007; Accepted 20 October 2007

\begin{abstract}
Adduct formation constants have been determined by spectrophotometric study of a few typical bases with nickel(II) chelate of 4-methyl-8quinolinol in chloroform. Bathochromic and hypsochromic shifts were observed in the visible range due to the adduct formation. Monobasic bases like pyridine, methyl substituted anilines and such other bases exhibit 1: 2 stoichiometry giving hexa - coordinated adductds . The dibasic bases such as 1,10-phenanthroline and 2,9- neocuproine exhibit $1: 1$ stoichiometry giving hexacoordinated adducts. The experimental results are discussed in terms of the basicity and steric effects of the various bases.
\end{abstract}

Keywords: Adduct formation, Steric hindrance, Formation constant.

\section{Introduction}

Nickel(II) chelates of several ligands like diphenylcarbazone, diphenylthiocarbazone, 8-quinolinol and its substituted analogues produce intense absorption in the optical spectra, in the visible range, which is characteristic of the ligand itself . Structural changes occurred in the nickel(II) chelates on addition of nitrogen bases forms adduct which gives rise to profound spectral changes. Such bathochromic and hypsochromic shifts observed in the visible region of the spectra of nickel(II) chelates of dithizone ${ }^{1}$, dipheylcarbazone ${ }^{2}$, 8-quinolinol ${ }^{3}$, methylsubstituted 8-quinolinols ${ }^{3}$, substituted diphenylcarbazones and dithizones $^{4-11}$ on addition of nitrogen bases. These were employed for the determination of adduct formation constants. The changes in spectra helps in confirming the structure of the 
nitrogen base adducts of nickel(II) chelates. Pyridine and its methyl derivatives were used to study the role of adduct formation in the extraction of $\mathrm{Zn}$ (II) and $\mathrm{Ni}$ (II) with substituted 8quinolinols ${ }^{12,13}$, dithizones ${ }^{14}$ and dipheylcarbazones ${ }^{15,16}$. Suresh et al. ${ }^{14-16}$ observed that by the addition of pyridine, 1,10-phenanthroline and 2,2 ${ }^{1}$ - bipyridyl to nickel(II) chelates of substituted dithizones and its analogues enhances the extraction of nickel into chloroform.

In this report, we give some results of study of the adducts of nickel(II)-4-methyl-8quinolinate (Ni4M8Q) with different nitrogen bases to gain the quantitative information about the nature and the stability of the adducts formed.

The adducts of Ni4M8Q have octahedral structures with monobasic and dibasic ligands. It may lead to better understanding of the behaviour of different donor atoms in the formation of adducts. All the reactions were carried out in chloroform as a solvent which does not add axially to the planar nickel(II) chelate.

\section{Experimental}

\section{Apparatus}

A Bausch and Lomb Spectronic 2000 UV- vis recording spectrophotometer and Systronic spectrophotometer 106 were used for the measurements of absorbance. Magnetic susceptibility measurements were carried out on the solids by Farady method ${ }^{17}$.

\section{Reagents}

The reagent 4-methyl-8-quinolinol was prepared as reported in the literature ${ }^{18}$. The product was purified by vacuum distillation (m.p. $141{ }^{\circ} \mathrm{C}$ ), Pyridine (Fisher), picolines (Eastman), lutidines, aniline, 2 Me-aniline (B.D.H.), 2, 3 Di Me-aniline (Seelge-Hannover), 2,6 Di Meaniline, (Loba), morpholine and piperidine (Merck) were dried over solid potassium hydroxide and distilled. Constant boiling fractions were collected and distilled. 1,10Phenanthroline, 2,9-neocuprione (G.Frederick Smith Co.), 4 Me-aniline (B.D.H.), nickel perchlorate (Fisher Reagent Grade) and chloroform (Merck) were used as received.

\section{Preparation of nickel (II)-4-methyl-8-quinolinate}

The nickel (II) -4-methyl-8-quinolinate was prepared by dissolving a known amount of the nickel perchlorate $(\sim 1 \mathrm{~g})$ in acetate buffer $(\mathrm{pH}=6.0)$ and mixing it with an appropriate amount of reagent in alcoholic solution. The total amount of reagent added was slightly more than that of required for 1:2 stoichiometry. The yellow precipitate formed was digested for $2 \mathrm{hr}$ and filtered hot. The complex was dried in vacuum over phosphorus pentoxide at room temperature. The nickel content of the complex was found to be $15.92 \%$ which is calculated by phenanthroline-dithizone method developed by Math et al. ${ }^{19}$ (Calc. $15.86 \%)$.

\section{Determination of equilibrium constants}

Known volume of chloroform solutions of Ni4M8Q were pipetted into standard flasks containing various amounts of the nitrogen base (dissolved in chloroform) and the mixtures were diluted to the mark with solvent. The absorption spectra of the solutions were obtained in the range 300-500 $\mathrm{nm}$, with an optical path length of $10 \mathrm{~mm}$. The absorbance value at 450 or $380 \mathrm{~nm}$ was found to be suitable for the study of formation of the Ni4M8Q adducts, The difference in absorbance between chelate and adduct being largest at these wave lengths. The absorbances were found to be constant for at least $1 \mathrm{hr}$, unless otherwise stated. Typical plot is shown in Figure 1. 


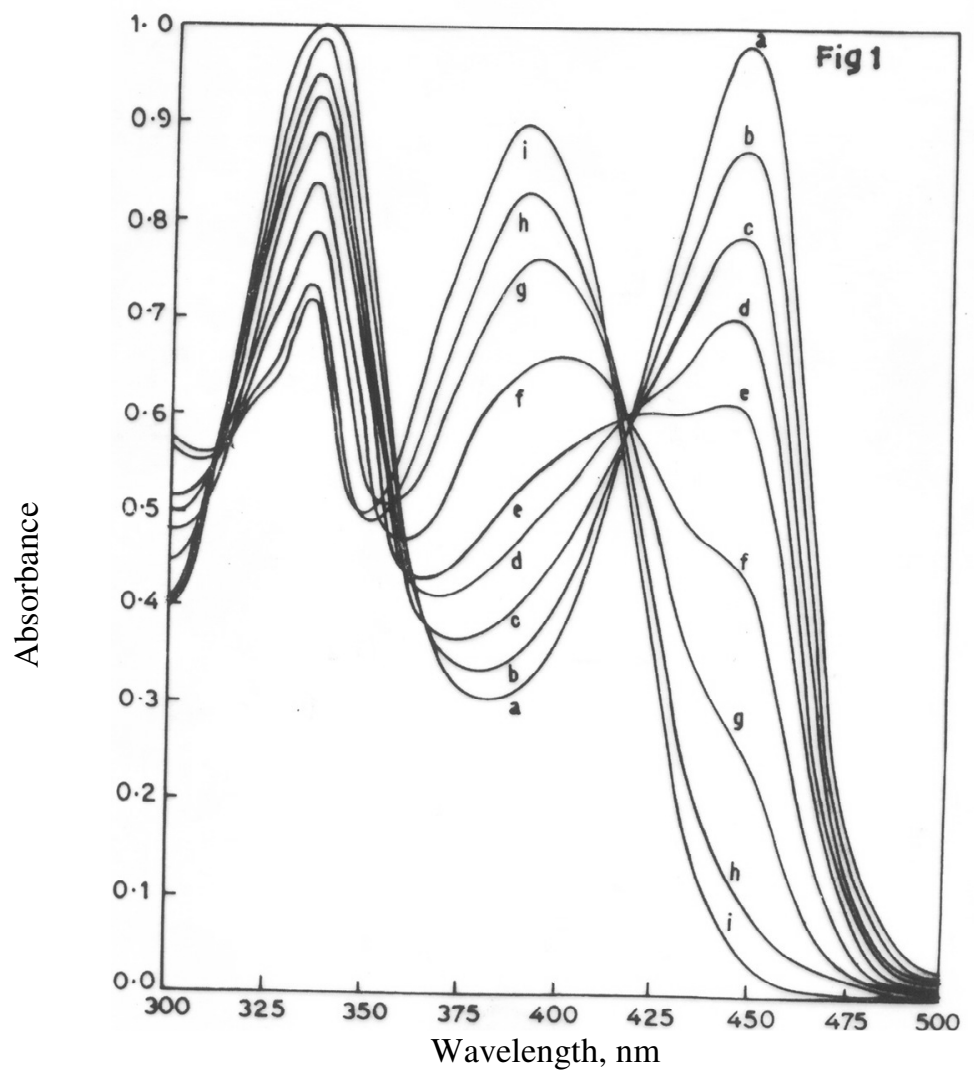

Figure 1.Absorption spectra of Ni4M8Q + 4-methyl aniline mixtures in chloroform at $25^{\circ} \mathrm{C}$. [NI4M8Q]: $12.10 \times 10^{-5} \mathrm{M}$, [4-Me-aniline] X $10^{2} \mathrm{M}$ : (a) 0.00 (b) 0.99 (c) 1.99 (d) 2.99 (e) 3.98 (f) $5.98(\mathrm{~g}) 7.97$ (h) 9.96 and (i) 11.95

\section{Results and Discussion}

The absorption spectra were measured in the visible region around $300-500 \mathrm{~nm}$ using chloroform as a reference at a concentration of $10^{-5} \mathrm{M}$. A typical spectrum for Ni4M8Q is shown in Figure 1. The light yellow coloured chloroform solution of Ni4M8Q has two bands in $450\left(\varepsilon_{\mathrm{mol}} \sim 8.09 \times 10^{4} 1 . \mathrm{mole}^{-1} . \mathrm{cm}^{-1}\right)$ and $340 \mathrm{~nm}\left(\varepsilon_{\mathrm{mol}} \sim 8.21 \times 10^{4} 1 . \mathrm{mole}^{-1} . \mathrm{cm}^{-1}\right)$ with two isosbestic points around 360 and $420 \mathrm{~nm}$ (Fig.1.). The addition of nitrogen bases to Ni4M8Q produced dark yellow coloured adduct having a single absorption band at $400 \mathrm{~nm}$. Measurements at wavelengths 450 and $380 \mathrm{~nm}$ gave similar results. However, the absorption at $450 \mathrm{~nm}$ only chelates absorbs which is suitable for analysis. Therefore, the absorption values at $450 \mathrm{~nm}$ was plotted as a function of $\mathrm{pB}$ to obtain sigmoidal curves. The values of $A_{a d}$ was obtained from the extrapolation of these curves which are used in the calculation of $\log \beta_{\mathrm{n}}{ }^{\text {ad }}$ using

$$
\log \beta_{\mathrm{n}}{ }^{\mathrm{ad}}=-\mathrm{n} \log [\mathrm{B}]+\log \left[\mathrm{A}_{\mathrm{ch}}-\mathrm{A} / \mathrm{A}-\mathrm{A}_{\mathrm{ad}}\right]
$$

where $\mathrm{n}$ is the number of base molecules, $\mathrm{B}$, attached to chelate; $\mathrm{A}_{\mathrm{ch}}$ and $\mathrm{A}_{\mathrm{ad}}$ are respectively, the absorbances due to chelate and adduct and $\mathrm{A}$ is the absorbance due to the chelate and adduct equilibrium mixture. 
In case of bidentate ligands, in addition to the slope of unity found from equation (1), the mole-ratio ${ }^{20}$ and Job's method ${ }^{21}$ were also used to confirm the ratio of base to chelate. The experimental data is given in Table 1 .

Table 1. Adduct formation constants of nickel(II)-4-methyl-8- quinolinate with nitrogen bases in chloroform

\begin{tabular}{|c|c|c|c|}
\hline Base & $\mathrm{pKa}$ & Slope(n) & $\log \beta_{\mathrm{n}}{ }^{\mathrm{ad}}$ \\
\hline Pyridine & $5.20^{+}$ & 2 & 3.48 \\
\hline 2-Picoline & $5.90^{+}$ & 2 & -0.12 \\
\hline 4-Picoline & $6.08^{+}$ & 2 & 3.82 \\
\hline 2,6-Lutidine & $4.95^{+}$ & 2 & 1.54 \\
\hline 2,4-Lutidine & $6.72^{+}$ & 2 & 2.32 \\
\hline Aniline & $4.63^{*}$ & 2 & 1.74 \\
\hline 2 Me-aniline & $4.44^{*}$ & 2 & 1.02 \\
\hline 4 Me-aniline & $5.08^{*}$ & 2 & 1.32 \\
\hline 2,3 Di Me-aniline & $4.64^{* *}$ & 2 & 1.26 \\
\hline 2,6 Di Me-aniline & $4.10^{* *}$ & 2 & 0.86 \\
\hline 2-Nitroaniline & & & NAF \\
\hline 3-Nitroaniline & & & NAF \\
\hline 4-Nitroaniline & & & NAF \\
\hline 1,10-phenanthroline & $4.95 *$ & 1 & 5.90 \\
\hline 2,9- Neocuproin & $5.85^{*}$ & 1 & 2.96 \\
\hline Morpholine & $8.33^{*}$ & 2 & 2.46 \\
\hline Piperidine & $11.12^{*}$ & 2 & 2.88 \\
\hline Ethyl alcohol & & 2 & 1.18 \\
\hline
\end{tabular}

The stabilities of nickel(II) adducts with monodentate bases, increases with pKa for the conjugated acid of the base. These adducts contain two moles of bases with slope of 2 for the plots of $\log \left[\mathrm{A}_{\mathrm{ch}}-\mathrm{A} / \mathrm{A}-\mathrm{A}_{\mathrm{ad}}\right]$ vs $-\log$ [Base]. This accounts for six coordination sites around nickel. The formation of such six-coordinate nickel(II) chelates has also been observed in the case of oxygen containing ligands ${ }^{4,10,11}$

The stabilities of Ni4M8Q - adducts were found to increase in the following order of bases: 2-picoline $<2,6 \mathrm{Di} \mathrm{Me}$-aniline $<2 \mathrm{Me}$ - aniline $<2,3 \mathrm{Di} \mathrm{Me}$ - aniline $<4-\mathrm{Me}$ - aniline $<2,6$-lutidine $<$ aniline $<2$,4-lutidine $<$ morpholine $<$ piperidine $<2$,9-neocuprion $<$ pyridine $<4$-picoline $<1,10$-phenanthroline. The lower stabilities of 2-picoline, 2,6 Di Me - aniline, 2Me - aniline, 2,6 Di Me - aniline and 2,9-neocuprion may be attributed to steric hindrance offered by methyl groups present at nitrogen donor group even though their $\mathrm{pKa}$ values are much more higher than pyridine, aniline and 1,10-phenanthroline. The high values of log $\beta_{\mathrm{n}}{ }^{a d}$ for nitrogen heterocyclic bases ${ }^{22,23}$ are compared to low values of $\log \beta_{\mathrm{n}}{ }^{\text {ad }}$ for aniline bases which indicate that anilines are probably the weaker bases. It may be anticipated ${ }^{24}$ that the stronger the basic character the stronger will be the bonding in Ni4M8Q - nitrogen base adducts. Hence it may be concluded that Ni4M8Q - pyridine adducts have stronger bonding than Ni4M8Q - aniline adducts as evident from $\log \beta_{\mathrm{n}}{ }^{\text {ad }}$ values in Table 1.

The smaller formation constants of the adducts of Ni4M8Q with morpholine may be attributed due to decrease in basicity of the nitrogen atom in the morpholine ring. From the study of adduct formation of Ni4M8Q with saturated heterocyclic nitrogen bases, it is clear that the structural and electrostatic rearrangements in the donor, as well as, in the acceptor molecules must be considered in the interpretation of adduct formation ${ }^{25}$. Fernando ${ }^{26}$ has 
shown that there is considerable distortion in nickel(II) chelates when these adducts are formed with stronger nitrogen bases. The donor molecules themselves have undergone structural changes $^{27}$, for example piperidine, in chair configuration, the hydrogen on nitrogen atom in the equatorial position. When the adduct formation occurs the hydrogen atom probably shifts to the axial position to allow the nickel atom to bond to the nitrogen and occupy the equatorial position.

Polar solvent like ethyl alcohol also form stable adduct like nitrogen bases. It is surprising to find that the absorption spectrum of Ni4M8Q does not change upon the addition of 2,3 and 4-nitroanilines. This may be due to the fact that these ligands do not form adducts with Ni4M8Q under these experimental conditions.

It is, however, interesting to note that, the adduct formation constants for Ni4M8Q are significantly higher to its sulphur and nitrogen nickel(II) chelates which indicates that, the adducts of nickel(II) chelates ${ }^{4,5}$ with $\mathrm{O} \& \mathrm{~N}$ atoms prefer higher coordination number than four.

\section{References}

1. Math K S and Freiser H, Anal. Chem., 1969, 41, 1682.

2. Suresh T, Dheerendra T and Kulkarni V H, Acta Cienca Indica, 1992, 18C, 113.

3. Bhatki K S, Rane A T and Freiser H, Inorg. Chim. Acta, 1978, 26, 183.

4. Math K S and Suresh T, Talanta, 1985, 32, 811.

5 Math K S, Suresh T and Aminabhavi T M, Spectrochemica Acta, 1986, 42A, 693.

6. Suresh T, Math K S and Kulkarni V H, Asian J. Chem., 1993, 5, 296.

7. Suresh T, Kulkarni V H and Hippargi S S, Nat. Acad. Sc. Letters, 1994, 17, 141.

8. $\quad$ Suresh T and Basavaraj B, Proc. Nat. Acad. Sc. India, 1998, 68A, 121.

9. Suresh T and Biradar V and Kulkarni V H, J. Chemtracks, 1999, 01, 1.

10. Suresh T, Kottureshwara N M and Suresh, J. Ind. Counc. Chem., 2004, 21, 38.

11. Suresh T, Nagesh Shastry D, Revanasiddappa M and Suresh, J. Ind.Chem. Soc., 2006, 83, 1.

12. Fa-Chun Chou and Freiser H, Anal. Chem., 1968, 40, 34.

13. Bhatki K S, Rane A T and Freiser H, Inorg. Chem., 1978, 17, 2215.

14. Suresh T, Acta Cienca Indica, 1994, XXC, 58.

15. Suresh T and Kulkarni V H, J. Ind. Chem. Soc., 1995, 72, 887.

16. Suresh T, J. Ind. Coun. Chem., 2002, 19, 47.

17. Landott - Bornstein, Zahlenwerte Und Funktionen (Magnetic Properties II), Springer-Verlag, Berlin, 1965.

18. Phillips J D, Elbinger R L and Merritt L, J. Amer. Chem. Soc., 1949, 71, 3987.

19. Math K S, Fernando Q and Freiser H, Anal. Chem., 1964, 36, 1762.

20. Joe J A and Jones A L, Ind. Eng. Chem., Anal. Ed., 1944, 16, 11.

21. Job P, Anal. Chim. Phys, 1928, 9, 113.

22. Suresh T, Ph.D. Thesis, Karnataka University, Dharwad, India, 1985.

23. Suresh T and Kottureshwara N M, Gulbarga University Research Journal (Sc. \& Tech.), Vijana Ganga, 2005, 04, 18.

24. Albert A and Serjeant E P, The Determination of Ionization Constants, Chapman and Hall Ltd., London, 1971.

25. Marshall T and Fernando Q, Anal. Chem., 1972, 44, 1346.

26. Fernando Q, Separ. Sci., 1969, 01, 575.

27. Gartio A F and Wayland B B, J.Amer.Chem. Soc., 1969, 21, 866.

28. Robert C Weast, Hand Book of Chemistry and Physics, Cleveland, Ohio, 1976.

29. Pollock J R A and Stevens R, Dictionary of Organic Compounds, Chlor-Dim, London 1965. 


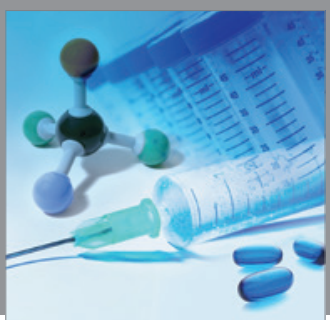

International Journal of

Medicinal Chemistry

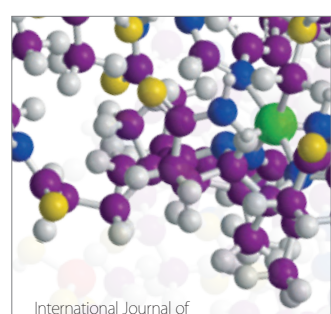

Carbohydrate Chemistry

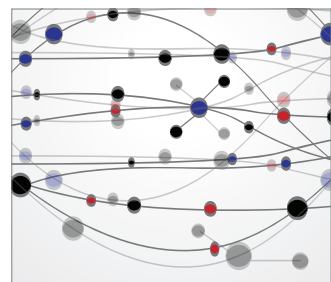

The Scientific World Journal
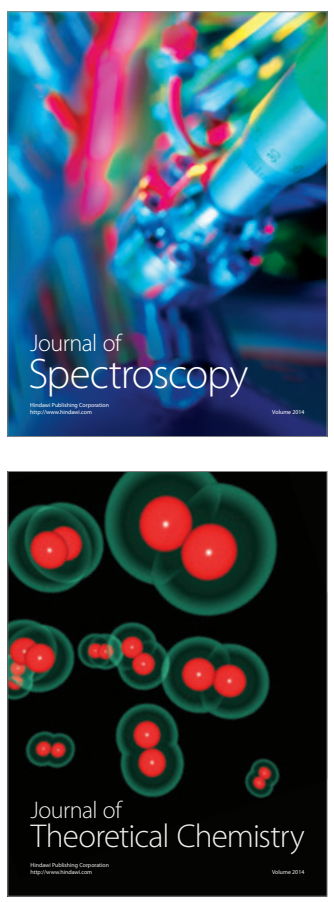
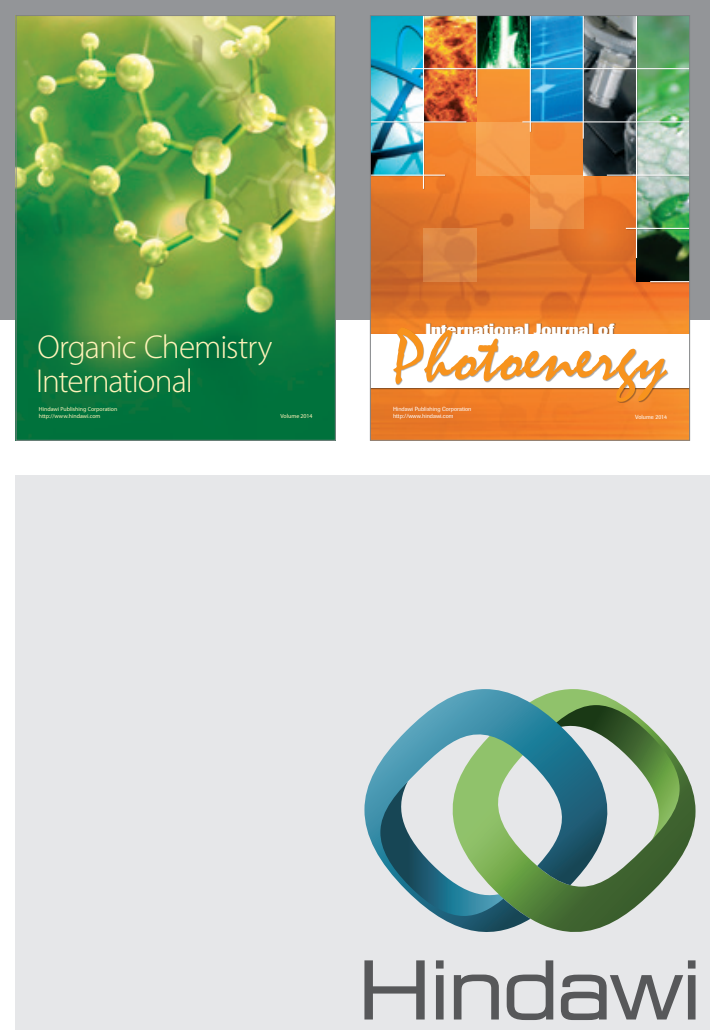

Submit your manuscripts at

http://www.hindawi.com
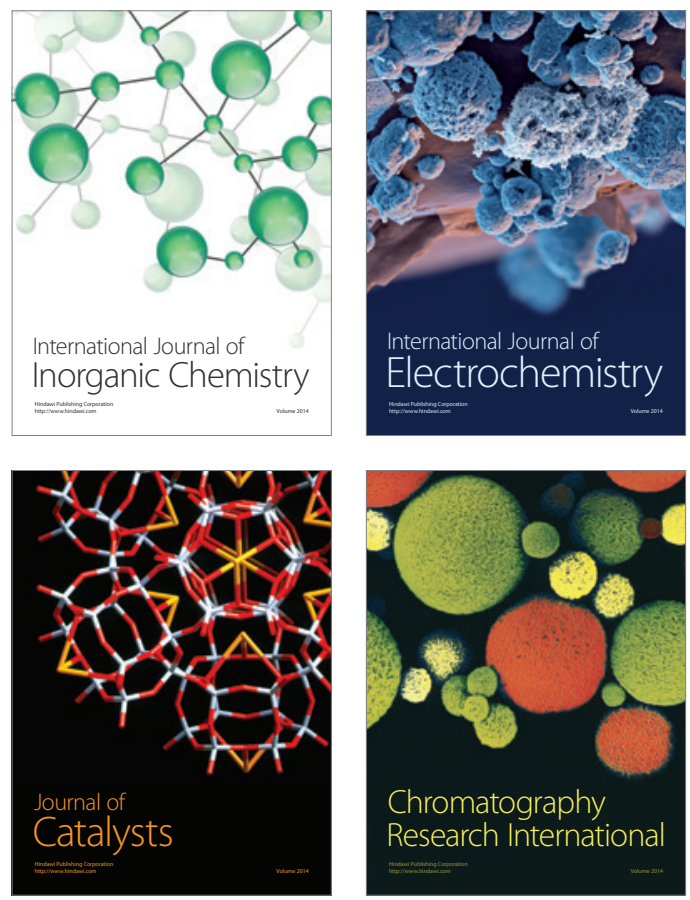
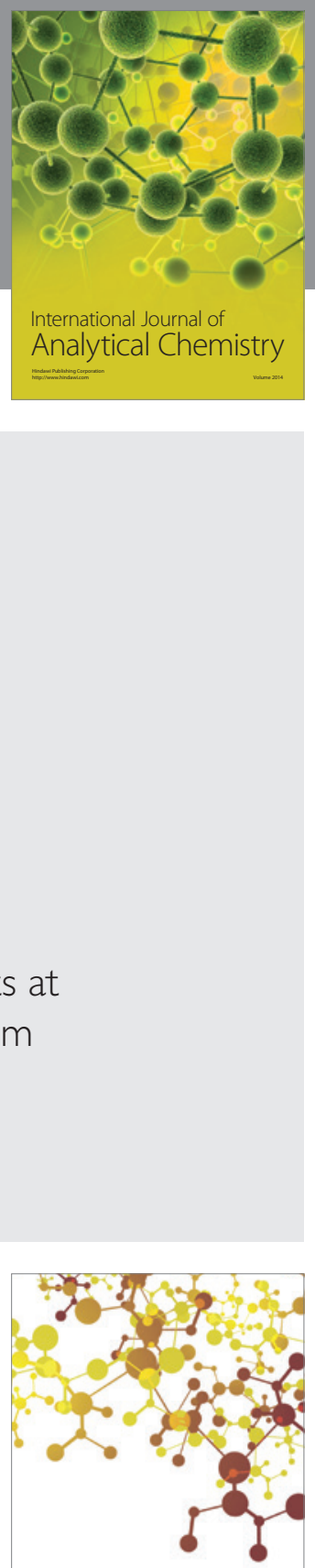

Journal of

Applied Chemistry
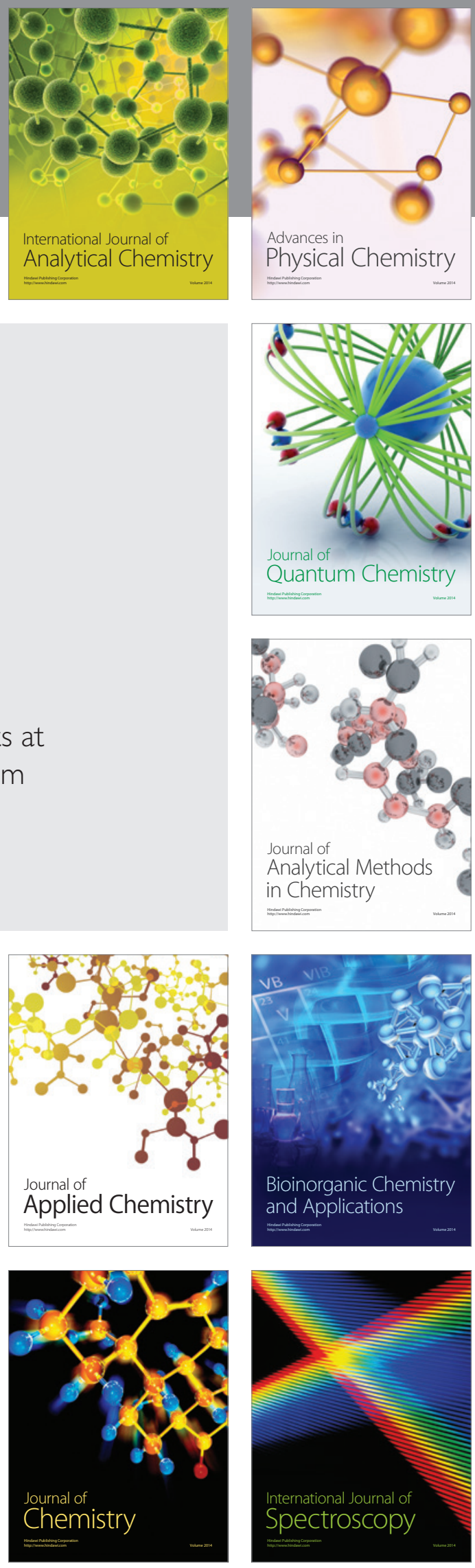